\title{
The spatial scaling of species interaction networks
}

\author{
Nuria Galiana', Miguel Lurgi', Bernat Claramunt-López ${ }^{2,3}$, Marie-Josée Fortin ${ }^{4}$, Shawn Leroux ${ }^{5}$, \\ Kevin Cazelles ${ }^{6,7,8}$, Dominique Gravel ${ }^{9}$ and José M. Montoya ${ }^{1 *}{ }^{1 \star}$
}

\begin{abstract}
Species-area relationships (SARs) are pivotal to understand the distribution of biodiversity across spatial scales. We know little, however, about how the network of biotic interactions in which biodiversity is embedded changes with spatial extent. Here we develop a new theoretical framework that enables us to explore how different assembly mechanisms and theoretical models affect multiple properties of ecological networks across space. We present a number of testable predictions on network-area relationships (NARs) for multi-trophic communities. Network structure changes as area increases because of the existence of different SARs across trophic levels, the preferential selection of generalist species at small spatial extents and the effect of dispersal limitation promoting beta-diversity. Developing an understanding of NARs will complement the growing body of knowledge on SARs with potential applications in conservation ecology. Specifically, combined with further empirical evidence, NARs can generate predictions of potential effects on ecological communities of habitat loss and fragmentation in a changing world.
\end{abstract}

T he species-area relationship (SAR) is among the most widely recognized ecological patterns ${ }^{1-3}$. The larger the geographical area sampled, the richer the ecological community ${ }^{2-4}$. SARs have been used extensively to estimate species richness in a given region $^{2,4,5}$ and to predict species extinctions due to habitat loss ${ }^{6-8}$. Yet, for several logistical reasons, most studies of SARs have been traditionally limited to particular taxa and functional groups. SARs for multi-trophic communities are just starting to be documented $^{9-11}$, along with the role played by biotic interactions in shaping these relationships ${ }^{10,12}$.

Biotic interactions modulate the outcomes of community assembly and disassembly. Different spatial processes in turn determine which interactions will be realized, ultimately regulating community dynamics $^{13-16}$. For example, higher dispersal rates of species at the top of the food web can increase the proportion of top predators in local communities and in turn enhance top-down regulation ${ }^{14,17}$. The relationship between area and biodiversity is thus inherently affected by the way ecological interactions and the emerging network structure of multispecies communities change according to the location and size of the area sampled. Unveiling the mechanisms underlying the relationship between area and biotic interactions will provide insights on ecosystem organization across spatial scales ${ }^{10,15,18-21}$.

Gaining a deeper understanding of network-area relationships (NARs) is arguably as important as the knowledge we have on SARs. Indeed, understanding the mechanistic basis of the spatial scaling of network properties is essential to predict the effects of disturbances such as habitat loss and fragmentation on the organization of multispecies communities, ultimately affecting their persistence and functioning. Disentangling how network structure changes with spatial scale is crucial to interpret empirical data on ecological networks. If the spatial scale affects network structure, then comparative studies should explicitly consider the area sampled as well as the environmental conditions to generate meaningful conclusions, as is systematically done in studies on diversity distribution patterns ${ }^{22}$.
Here we propose NARs as a theoretical and predictive framework to study the variation of the properties of ecological networks (for example, connectivity, trophic level composition, trophic chain length) across spatial scales, from small to large areas. We first showcase a number of spatial processes (for example, dispersal) that could generate different types of NAR. Then we present three simple theoretical models to understand and test how NARs could emerge given specific processes of spatial assembly of multi-trophic communities. As such, we provide new insights on the role of spatial processes in community assembly and structure, and explain how these can be used to predict the effects of habitat loss and fragmentation on not only species richness across trophic levels, but also the structure of biotic interactions. Last, we propose further theoretical and empirical research avenues stemming from our NARs framework that could contribute to a unified theory of the spatial scaling of ecological communities.

\section{Mechanisms behind NARs}

There are several possible mechanisms responsible for changes of network structure across spatial scales. Figure 1 provides a synthesis of those analysed here, our expectations for the emergence of NARs based on three mechanisms and the theoretical models used to evaluate our expectations.

The first mechanism is derived from the SAR. There is an associated increase in the number of interactions (links) with the increase of species richness with area. Two major hypotheses have been proposed to account for the variation of the number of links with species richness in food webs. Both hypotheses do not explicitly account for SARs. The link-species scaling law ${ }^{23}$ states that species interact with a constant number of species independently of species richness ${ }^{24,25}$. In contrast, the 'constant connectance hypothesis ${ }^{26}$ states that the fraction of potential interactions realized (that is, the number of trophic links, $L$, standardized by the number of potential interactions, $S^{2}$ ) is constant across food webs,

'Ecological Networks and Global Change Group, Theoretical and Experimental Ecology Station, CNRS and Paul Sabatier University, Moulis, France. ${ }^{2} \mathrm{CREAF}$, Edifici Ciències, UAB, Bellaterra, Catalunya, Spain. ${ }^{3}$ Ecology Department, BABVE, Bellaterra, Catalunya, Spain. ${ }^{4}$ Department of Ecology \& Evolutionary Biology, University of Toronto, Toronto, Ontario, Canada. ${ }^{5}$ Department of Biology, Memorial University, St. John's, Newfoundland and Labrador, Canada. ${ }^{6}$ Département de Biologie, Chimie et Géographie, Université du Québec à Rimouski, Quebec, Canada. ${ }^{7}$ Quebec Center for Biodiversity Science, Montreal, Quebec, Canada. ${ }^{8}$ UMR MARBEC, Université de Montpellier, Montpellier, France. ${ }^{9}$ Département de Biologie, Université de Sherbrooke, Sherbrooke, Quebec, Canada. *e-mail: josemaria.montoyateran@sete.cnrs.fr 


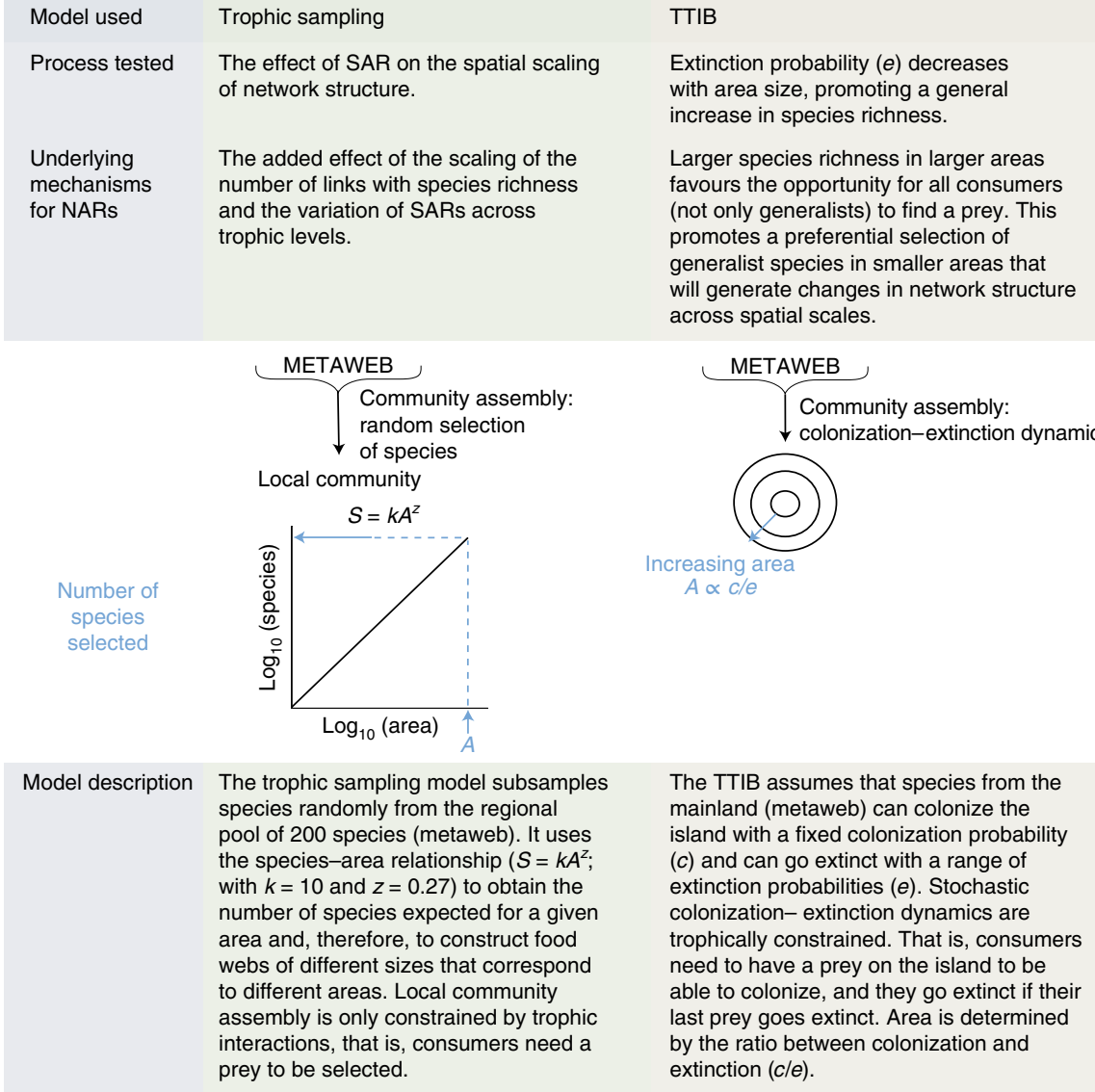

\section{Trophic meta-community}

Dispersal limitation increases spatial heterogeneity in species composition (that is, beta-diversity)

The higher the heterogeneity in species composition across space, the stronger the dependency of network structure with area: increasing the area sampled increases the number of new species sampled and has a stronger effect on network properties.

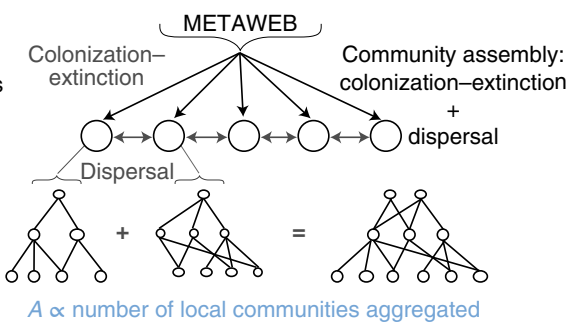

It consists of 75 local patches connected to the mainland (metaweb) and to each other, depending on the distance. Species can arrive at each local patch from the metaweb with a fixed colonization probability and can go extinct with a fixed probability. Species can disperse between connected local patches according to different dispersal values. Stochastic colonization-extinction dynamics and dispersal are trophically constrained. Area is determined by the aggregation of local communities (patches) in an ever-increasing fashion from 1 to the maximum number of communities (75).

Fig. 1 | A mechanistic approach to understand NARs.

irrespective of species richness. Empirical evidence suggests that link-species richness relationships lie between the two hypothe$\operatorname{ses}^{27,28}$. If we introduce area within these link-species scaling hypotheses, given that $S$ increases with area and $L$ scales with $S$, we expect changes in food web structure with area simply emerging from SAR that are in turn shaped by the specific link-species relationship in place. A previous study ${ }^{18}$ proposed a scaling of trophic links with area by combining species-area and the link-species scaling theories mentioned above. Following the authors' approximation, we generated trophic communities of different sizes (that is, different number of species) with the trophic sampling model. This model randomly subsamples species from the metaweb (that is, food web of 200 species generated with the niche model $^{29}$; see Supplementary Methods and Supplementary Table 1 for a full description), which conforms the regional pool of species, with the only constraint that each consumer needs at least one prey to be selected (Fig. 1). We expect different shapes of the species richness-area relationships at each trophic level emerging from this trophic constraint. If the spatial scaling of species richness differs among trophic levels, different facets of network structure are expected to change with area. As a consequence, the proportion of species belonging to each trophic level (for example, basal, intermediate and top species) will be different on each spatial scale, triggering further consequences on community structure ${ }^{9,10,30}$. We explore the combination of both mechanisms (that is, the scaling of the number of links with species richness and the variation of SARs across trophic levels) with the trophic sampling model (Fig. 1; Supplementary Methods).

The second mechanism arises from the scaling of colonizationextinction dynamics in multi-trophic communities with area. This was first considered in the theory of island biogeography ${ }^{4}$ (TIB) and its extension to trophic interactions ${ }^{16,30-32}$ (trophic theory of island biogeography, TTIB). The TIB predicts the richness of local assemblages from the equilibrium between colonization and extinction processes. It assumes that the closer the island is to the mainland the larger the colonization rate ${ }^{4}$, and that the larger the island size the lower the extinction rate due to the increase in population sizes $^{33,34}$. The TTIB incorporates a trophic constraint not considered in the TIB: consumers must have a prey on the islands they colonize to be able to establish and persist. Therefore, the richness of the local assemblage and their biotic interactions are defined by the equilibrium between colonization and extinction processes, where species that are diet generalists and/or belong to lower trophic levels are preferentially selected given that they are less affected by the trophic constraint. Generalist species have been shown to be faster colonizers than specialists, ultimately shifting community structure through time ${ }^{16,35}$. We expect stronger impact of this trophic constraint at smaller areas, where the number of species is smaller. As area increases, the number of species also increases, which in turn increases the opportunity for consumers to find a prey, and therefore not only generalist species will be able to colonize, but also specialists, ultimately promoting changes in network structure as area changes. We use the TTIB mode ${ }^{16}$ to generate islands of different sizes based on different colonization/extinction ratios, where colonization rate is fixed to analyse the network structure resulting from the assembly process for each island size (Fig 1; Supplementary Methods).

The third mechanism arises from the spatial variability in community composition, that is, spatial turnover of species. Clumping of species underlies beta-diversity and SARs ${ }^{36,37}$. Its effect on the variation of network structure with area is driven by the increase in the number of species and their interactions as area increases. 
The spatial turnover of species composition can be explained by several processes ${ }^{38}$. Here we focus on the spatial configuration and connectivity of the landscape, which ultimately determines the rates of dispersal of organisms between sites. In fragmented landscapes with major dispersal barriers, species turnover is higher than in homogeneous and continuous environments ${ }^{39}$. Changes in network structure with area are likely to be mediated by dispersal limitation through its effects on spatial turnover. Larger beta-diversity values will generate larger changes in network structure with area, because the number of different species encountered as the area sampled increases will be larger. To test the effects of this process we employ a multi-trophic meta-community model, extending the above-mentioned TTIB to entire landscapes, where we control species dispersal between local patches (Fig 1; Supplementary Methods).

\section{Multi-trophic community assembly models}

We explore the effects of each process on the spatial scaling of food web structure with the three models of multi-trophic community assembly mentioned above. We then analyse several network properties on different spatial scales, which allows us to characterize a suite of NARs (see Supplementary Methods for a full description of the assembly models and the network properties used). In this section, we first present the emergence of the mechanisms tested for each assembly model and its effects on the spatial scaling of food web structure. We then provide a comparison between the predictions emerging from each model.

Trophic sampling model. Mechanisms. The number of links scales exponentially with species richness (slope $=1.91 \pm 0.003,95 \%$ confidence interval, in log-log space; Fig. 2a). Although the relationship falls between the two link scaling hypotheses (link-species scaling law, slope $\approx 1$ in log-log space; and constant connectance hypothesis, slope $\approx 2$ in log-log space), our results better support the latter. Additionally, we observe different SARs across trophic levels (Fig. 2b). The number of intermediate species increases significantly faster with area than the number of top and basal species
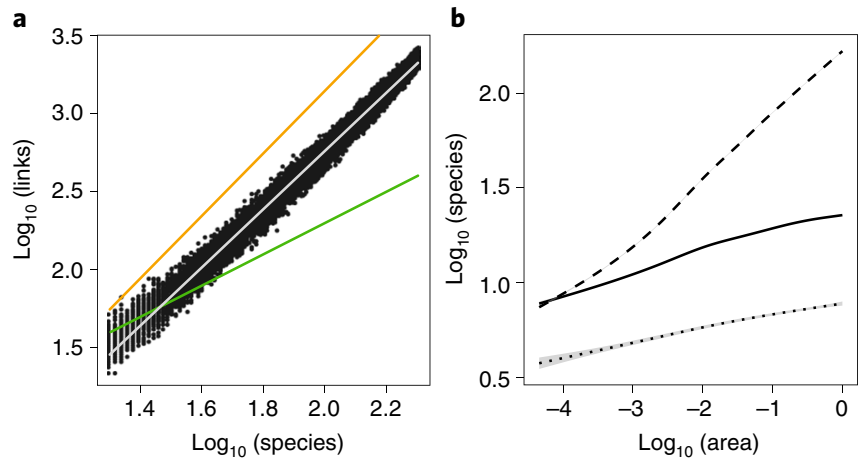

Fig. 2 | Mechanisms underlying NARs. a, Scaling of the number of links with species richness. Orange line, constant connectance hypothesis $(\mathrm{CCH}$; that is, the number of links in a web increases approximately as the square of the number of trophic species: $L \approx S^{2}$ ); green line, link-species scaling law (LSSL; the number of links per species in a web is constant and scale invariant at roughly two: $L \approx 2 S$ ); grey line, links-species relationship for the trophic sampling model. b. SARs per trophic level for the trophic sampling model. Area values close to -4 correspond to local communities and values close to 0 correspond to regional communities. Notice that area was rescaled to fall in the range between 0 and 1 , where 0 is the smallest local scale and 1 is the largest regional scale, and these are the log-transformed values of area. Black line, basal species; dashed line, intermediate species; dotted line, top species. Shaded areas correspond to $95 \%$ confidence intervals. (see Supplementary Table 2 for statistical analyses). Taken together, these results show that both mechanisms suggested as possible drivers of NARs - link scaling and different shape of the SARs across trophic levels - are at play in the trophic sampling model. As expected, these mechanisms trigger changes in network structure from local to regional scales.

NARs. Network complexity properties smoothly increase with area (Fig. 3a, Supplementary Fig. 1 and Supplementary Table 3). Whereas number of species, links per species, mean indegree and mean outdegree (that is, mean generality and mean vulnerability, respectively) show a pronounced sub-linear increase quickly approaching the asymptotic value set by the regional network, total number of links increases linearly with area. As a consequence, owing to its quadratic relationship with the number of species $(C=$ $\left.L / S^{2}\right)$, connectance decays sharply with area. That is, network complexity increases with area because larger areas have more species, more links and more links per species. However, given the faster rate of increase in the number of species than in the number of links, we observe a decrease in connectance. Network vertical diversity properties increase with area (Fig. 3b, Supplementary Fig. 1 and Supplementary Table 3). Mean food chain length (MFCL), fraction of omnivory and fraction of intermediate species increase sharply with area, reaching the asymptote corresponding to the regional values at relatively small areas. This, in turn, decreases asymptotically the fraction of basal and top species with area. Notice, however, that the fraction of herbivores (included within the category of intermediate species) decreases asymptotically with area, in parallel to the decrease on the fraction of basal species (Supplementary Fig. 2).

Unexpectedly, network modularity and the distribution of food web motifs do not show strong variations across spatial scales (Fig. 3d and Supplementary Fig. 1). Modularity, the proportion a

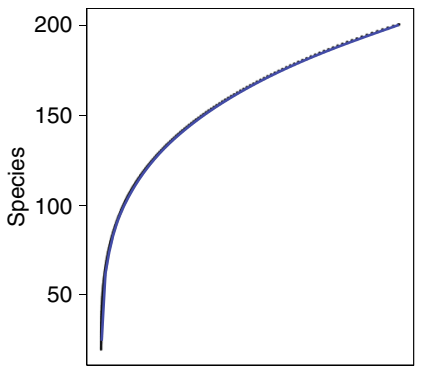

C

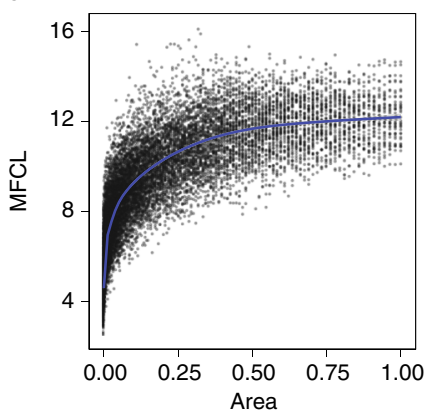

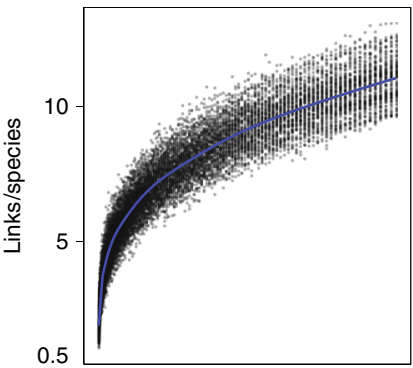

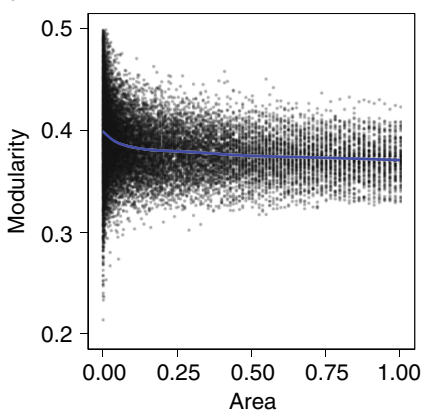

Fig. 3 | NARs for the trophic sampling model. Area values close to 0 correspond to local communities and values close to 1 correspond to regional communities. a-d, Relationship of the number of species (a), the number of links per species (b), Mean Food Chain Length (MFCL) (c) and modularity with area (d). Notice that the SAR shown in $\mathbf{a}$ is given by $S=k A^{z}$, where $A$ is area, $k=10$ is a constant and $z=0.27$ is the scaling factor of the number of species $(S)$ with area. Lines represent a generalized additive model fitted to data points. 
of simple chains, and apparent competition slightly decrease with area, while the proportion of exploitative competition shows a small increase with area.

TTIB model. Mechanisms. The proportion of specialist species increases with area (Fig. 4a,b). This indicates that species feeding on a larger number of prey do persist better in small patches than specialist species. Potential indegree distributions (quantified as the species indegree in the metaweb) are consequently shifted towards smaller values with increasing area (Fig. 4b). Interestingly, this preferential selection of generalist species on smaller scales does not affect the shape of the realized cumulative indegree distributions of the local networks (Fig. 4c). Independently of island size, indegree distributions are skewed, that is, there are more specialist than generalist species in all networks regardless of area. However, it is important to notice that the most specialized species (indicated with arrows in Fig. 4c) on small islands have more prey (that is, they are more generalist) than the most specialized species on large islands. In other words, at smaller areas, we observe a preferential selection of species that are generalists in the regional pool. As area increases, more specialized species are able to persist, which manifests both in a reduction in mean potential indegree and higher specialization of the most specialized species.

NARs. TTIB predictions do not differ qualitatively from the trophic sampling model. All facets of network complexity increase with area sub-linearly (Supplementary Fig. 3 and Supplementary Table 3), except for connectance, which decreases with area. As for the trophic sampling model, the faster rate of increase in the number of species than in the number of links causes the decrease in network connectance, even though the number of links per species also increases. In terms of vertical diversity, we observe a sharp increase in omnivory, MFCL and fraction of intermediate species (but see Supplementary Fig. 2), whereas the fraction of basal and top species show a drastic drop with increasing area (Supplementary Fig. 3 and Supplementary Table 3). Network properties of communities assembled with the TTIB model show more abrupt changes with area than the trophic sampling model, with asymptotes of all food web properties reached at smaller areas. The difference between the
TTIB and the trophic sampling models lies in the complexity of the assembly process. The stochastic nature of the community assembly enforced by the TTIB model, which is the result of colonizationextinction dynamics, favours the persistence over time of generalist consumers. Specialist consumers that depend on a single resource are more prone to become secondarily extinct given the trophic constraint: if their only resource goes stochastically extinct, they go extinct too. In contrast, the trophic sampling model only searches for possible configurations of a given number of species where every consumer needs to have a resource, without subjecting the selected community to additional stochastic extinctions. Therefore, the effects of the trophic constraint become more evident in the TTIB.

The modular structure and the distribution of motifs of the communities are again not strongly affected by the spatial scale (Supplementary Fig. 3 and Supplementary Table 3). Modularity is constant across spatial scales. However, the proportion of simple chains and apparent competition slightly decrease with area, whereas the proportion of exploitative competition increases.

Trophic meta-community model. Mechanisms. Dispersal limitation among local patches affects the turnover of species composition in our meta-communities. Beta-diversity decreases with dispersal rate (Supplementary Fig. 4), having further consequences for the spatial scaling of network structure. High dispersal rates increase local diversity (that is, scaled area 0 ) and reduce beta-diversity (Supplementary Fig. 4), making food webs more similar across the landscape. This implies that the amount of change in network structure is smaller, and that the asymptote that corresponds to regional network properties is reached at even smaller areas than for low values of dispersal. As a consequence, for high values of dispersal, we need to aggregate a smaller number of local communities to recover the structure of the large metaweb than with low dispersal rates and with the TTIB.

NARs. At low dispersal rates, NARs are similar to those observed for the TTIB. Both network complexity and vertical diversity change with area at a smaller rate compared with the high dispersal scenario (Supplementary Fig. 5 and Supplementary Table 3). High levels of dispersal among local communities weaken the scale-dependency of network structure: increasing the area sampled has less effect on

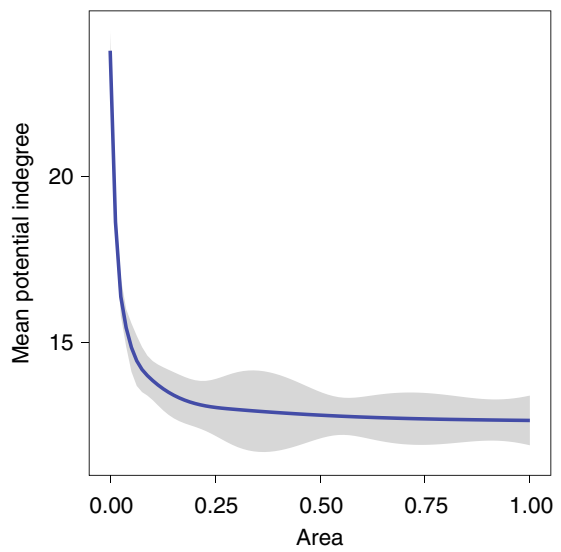

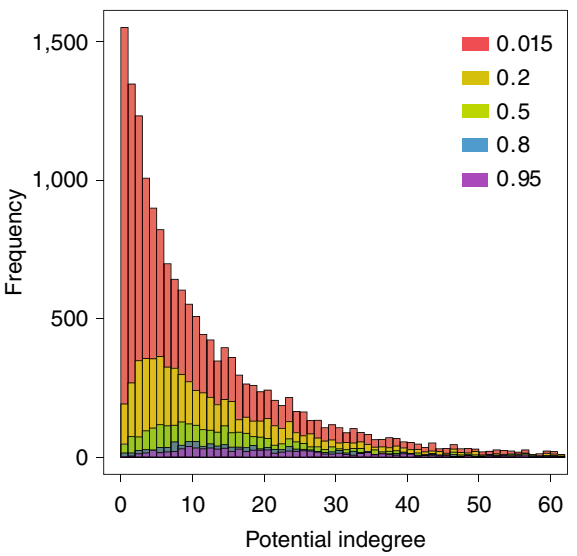

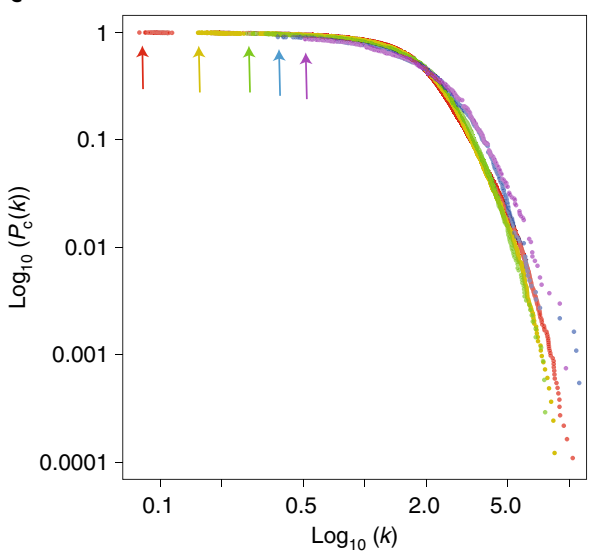

Fig. 4 | Specialism across spatial scales. a, Variation of food web mean potential indegree - quantified as the species indegree (that is, generality) in the metaweb - with area for the TTIB model. Area values close to 0 correspond to local communities and values close to 1 correspond to regional communities. Shaded areas show 95\% confidence intervals. b. Species potential indegree distributions for islands of different sizes simulated as the ratio between colonization and extinction rates, with higher ratios representing larger island areas. Colonization rate is fixed at 0.2 and each colour represents a different value of extinction (that is, red and purple correspond to the largest and the smallest area, respectively). c, Realized indegree distributions across spatial scales for the TTIB model. The cumulative probabilities $P_{c}(k)$, for $\geq k$, where $P(k)$ is the probability a species has $k$ prey in the network, is represented normalized by the mean number of links per species in the network. Coloured arrows show the starting point of the distribution for each island size. Colours correspond to the same area sizes as in $\mathbf{b}$. 
network properties at high levels of dispersal because the values of the properties of the regional network (that is, the asymptote) are reached earlier. Dispersal increases food web complexity (that is, more species, links and links per species) and its vertical diversity on both local and regional scales (Supplementary Fig. 5 and Supplementary Table 3).

As for the previous two models, network modularity and the distribution of motifs show small variations across spatial scales for both dispersal levels, being slightly less pronounced for high dispersal levels (Supplementary Fig. 5). Network modularity, the proportion of simple chains and apparent competition show a marginal decrease with area, while the proportion of exploitative competition shows a small increase.

Comparison between models. We found both quantitative and qualitative differences among NARs resulting from the three models (Fig. 5, Supplementary Table 3 and Supplementary Fig. 6). Qualitatively, the trophic sampling model shows smooth changes in network structure with area. The changes in network structure observed with the TTIB will be more abrupt as area increases, reaching the asymptote faster. Similarly, the trophic meta-community model with low dispersal leads to abrupt changes in network structure with area. However, important qualitative differences exist between the two that allow for determining the most likely mechanism behind empirical patterns of network scaling (Box 1, Supplementary Fig. 6). Finally, the trophic meta-community model with high dispersal shows the smallest change in network structure with area, reaching the asymptote for the regional network on very small spatial scales.

Quantitatively, the trophic sampling model shows the lowest rate of growth $(g$, measured as the steepness of a bounded exponential fitted to the data; Supplementary Table 3) for all complexity properties, followed by the trophic meta-community model with low dispersal, the TTIB and lastly the trophic meta-community model with high dispersal. This implies that increasing the area sampled has a less abrupt effect for NARs in the trophic sampling model, but this effect is manifested over a larger range of areas sampled. At the other extreme of the spectrum, the trophic meta-community model with high dispersal shows a rapid change in network structure on relatively small spatial scales. Hence, the scale-dependency of network structure depends on whether we focus on the rate of change of a given network property for a given increase in area, or on the range of areas across which the property changes.
The trophic meta-community model with low dispersal has the lowest growth rate for most vertical diversity properties, followed by the trophic sampling model. Whereas the TTIB shows the highest growth rate for the proportion of basal and intermediate species, the trophic meta-community model with high dispersal shows the highest values for the proportion of top species and MFCL (Supplementary Table 3). We compared each model with its non-trophic constrained version in Supplementary Fig. 7. The comparison shows a faster initial increase in complexity for communities assembled using the unconstrained versions of the TTIB and the trophic meta-community model with a levelling off for larger areas, while the unconstrained version of the trophic sampling model only shows differences for vertical diversity metrics (Supplementary Fig. 7).

\section{Testable predictions}

We presented a theoretical framework predicting the existence of a number of NARs in spatial multi-trophic communities, arising from different assembly processes. Although we obtained some universal predictions independent of the particularities of the assembly process used, we found differences in regard to the exact shapes of the specific NAR under scrutiny. This allows for specific predictions emerging from each model to be tested with empirical data (Boxes 1 and 2). In particular, we showed that the existence of different SARs across trophic levels has consequences for the variation of network structure with increasing area, the preferential selection of generalist species at small areas causes drastic changes on network structure in space and dispersal limitation is a key process influencing trophic interactions across spatial scales. Here we summarize and discuss a number of empirically testable predictions emerging from our framework (Boxes 1 and 2), and provide suggestions on where to focus future research efforts to better understand the causes and consequences of the variation of network structure across spatial scales.

\section{Multi-trophic SARs and NARs}

The spatial scaling of network structure cannot be fully explained by the increase in species richness with area. It is well established that species richness affects several food web properties. In many cases, differences in network properties simply result from differences in species richness between the communities studied ${ }^{40-43}$. However, the spatial scaling of species richness is likely to vary across trophic levels ${ }^{9,10,30}$. This differential scaling has further consequences for the variation of trophic network structure with area $\mathrm{a}^{10,12,30}$. A recent study $^{10}$ showed that the slope of the SAR steepens from plants to
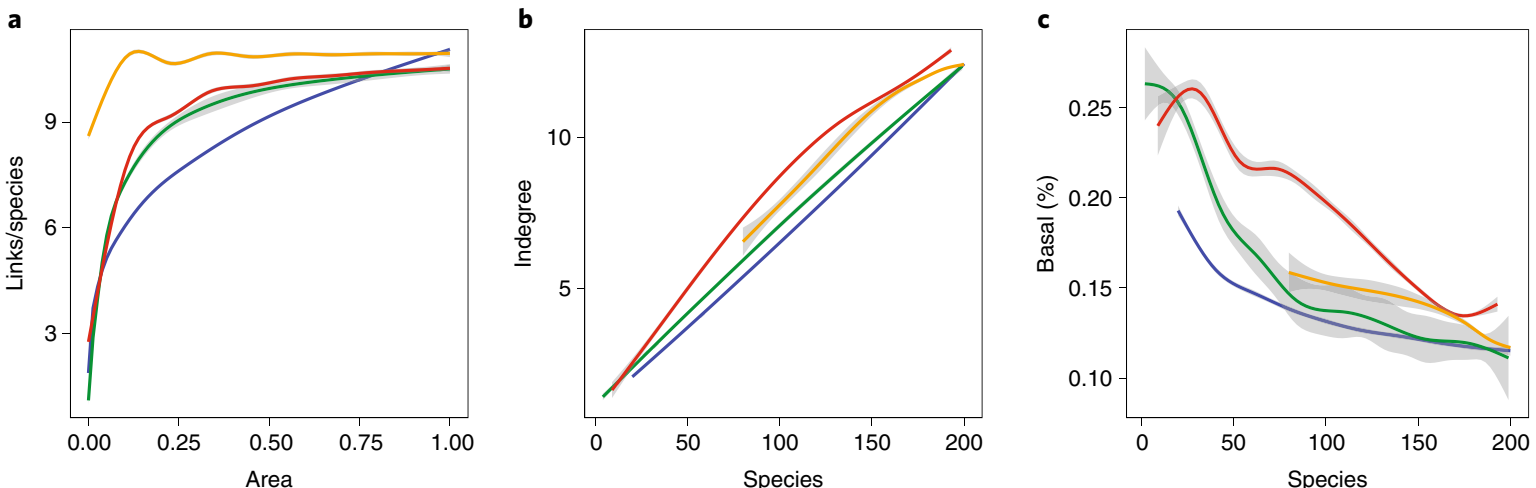

Fig. 5 I Comparison between models. a, Variation of the number of links with area for the three different models of community assembly. Area values close to 0 correspond to local communities and values close to 1 correspond to regional communities. $\mathbf{b}, \mathbf{c}$, Comparison between models controlling by the number of species. For a given number of species, differences in network properties between models. Blue line, trophic sampling model; green line, TTIB; red line, trophic meta-community model with dispersal $(d)=0.01$; orange line, trophic meta-community model $d=0.1$. Notice that high dispersal rates in the trophic meta-community model increase local diversity, resulting in local communities with more than 70 species. Lines represent a generalized additive model fit to data points. Shaded areas show $95 \%$ confidence intervals. 


\section{Box 1 | Empirical data and testable predictions}

Each model used could be represented by a different empirical dataset. The trophic sampling model (panel a below) corresponds to random subsamples of different area sizes all included within a larger homogeneous area. The TTIB model (panel b below) corresponds to independent and isolated islands (or patches) of different sizes (for example, an archipelago). The trophic metacommunity model (panel c below) corresponds to independent local communities (for example, patches, islands) of the same size connected through dispersal, where the spatial scaling of network structure is given by the progressive aggregation of different localities.

We can predict a different scaling of network structure in space emerging from each type of data (Fig. 5 and Supplementary Fig. 6). When area is subsampled randomly, network structure will smoothly change as we increase the size of the area sampled. In isolated islands, where each island constitutes one fully assembled community, changes in network structure will be more abrupt as area increases, reaching the asymptote faster. In metacommunities, the spatial scaling of network structure will be determined by the spatial heterogeneity in species composition. In fully connected metacommunities (that is, high dispersal), changes in network structure with area will be minimal because the complexity and the vertical diversity of the regional network are already reached on small spatial scales. In poorly connected metacommunities (that is, low dispersal), the spatial scaling of the majority of the network properties will be qualitatively similar to that observed in isolated islands.

However, two fundamental network properties can elucidate the difference between the two types of data: connectance and the percentage of basal species. In highly fragmented metacommunities with low dispersal, network connectance and the percentage of basal species will be higher through the entire range of areas (Supplementary Fig. 6), suggesting that the trophic constraint is having a strong effect across spatial scales. In isolated islands, connectance and the percentage of basal species drop drastically with a minimal increase of area, indicating a relief of the trophic constraint with area. $\mathbf{a}$

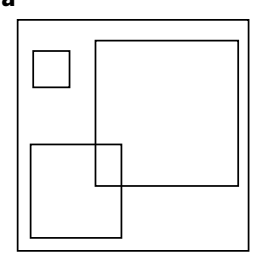

b

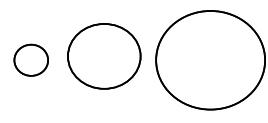

C

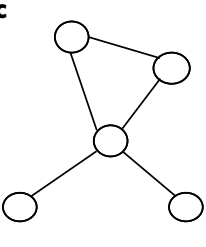

herbivores and from primary to secondary parasitoids. This in turn triggers a decrease in food chain length from large to small islands. In contrast, our trophic sampling model showed the steepest species-area slope for intermediate species (Fig. 2b). This contrasting result can be attributed to the fact that parasitoids tend to have exceptionally narrow diet breadths when compared with other top predators, being classified as a separate category within food webs in comparative analyses ${ }^{28}$. We considered wider diet breadths for top predators, which allowed them to overcome the trophic constraint $^{9,30}$ and therefore reduce the slope of their SARs by being selected locally, even when the number of species was small. The trophic sampling model thus shows that, in the absence of spatial structure, and in totally homogeneous communities, different SARs across trophic levels will emerge and will bias NARs towards higher fractions of intermediate species and longer food chains.

\section{Box 2 | Universal predictions}

Network complexity. Network degree distribution preserves its skewness across spatial scales, but specialism increases with area. Indegree distributions are skewed regardless of area, that is, there are more specialist than generalist species in all networks irrespective of the spatial scale (Fig. 4c). The preferential selection of generalist species on smaller scales affects the starting point of the distribution - determined by the most specialized species but not its shape. The most specialized species have more prey on smaller than on larger spatial scales.

Network vertical diversity. SARs vary across trophic levels. In food webs, the number of intermediate species increases faster with area than the number of top and basal species (Fig. 2b). This results in steeper slopes of SARs for intermediate species. Networks where top predators are heavily specialized, that is, host-parasitoid networks, should be an exception, with steeper slopes as trophic level increases.

The proportion of omnivorous links increases with area, promoting an increase of food chain length. The faster increase in the number of intermediate species with area facilitates the growth of the number of links among intermediate species (for example, intraguild predation), generating an increase of food chain length.

Network modules. Network modularity is constant across spatial scales in homogeneous landscapes. Heterogeneous landscapes, however, are likely to promote the emergence of network compartments due to, for example, the effect of species sorting. This is likely to generate an increase of modularity with area, as more compartments will be captured as the area sampled increases.

In the theoretical work developed in a previous study ${ }^{18}$, where the authors derived the spatial scaling of trophic links with area by combining the species-area relationship and the link-species relationship, they predicted the effect of having different SARs across trophic levels for the scaling of the number of links with area. Here, we extended the analyses of this effect to many other aspects of network structure beyond the number of links (for example, degree distributions, MFCL or modularity). By using a mechanistic approach to understand the spatial scaling of network structure we can determine the specific effects of each process tested, and generate specific and testable predictions on how network structure will change with area depending on the spatial scenario and the processes in operation.

In agreement with our expectations, the TTIB model exhibited a strong variation of network structure with area, mediated by the preferential selection of generalist species that emerges from the trophic constraint (Fig. 4). The effect of this constraint on species occupancy decreases with area because the total number of species increases, whereby the chances of finding a suitable prey also increase. Thus, colonization-extinction dynamics favoured greater occupancy of generalist consumers in small areas, where fewer prey are available. The occupancy for a given colonization and extinction rate is predicted to reach an asymptote with increasing prey species richness, because for larger diet breadths, consumers are no longer constrained to find their prey ${ }^{16,35}$. The comparison between the TTIB and its non-constrained TIB version shows a faster initial increase in complexity (that is, species and links/species) for communities assembled using the TIB with a levelling off for larger areas, illustrating the loss of importance of the trophic constraint as area increases (Supplementary Fig. 7). Therefore, as the area sampled increases, the proportion of specialist species also increases (Fig. 4a,b). 
Food web degree distributions are usually skewed (many specialists, few generalists $)^{44-46}$. In spite of the fact that smaller islands host species with larger potential diet breadth (that is, species indegree in the metaweb; Fig. 4a,b), the indegree distributions of the realized food webs kept this characteristic skewness (Fig. 4c). Given the importance of the degree distribution to community robustness to species loss, this suggests that food web robustness is preserved across spatial scales. The TTIB thus suggests that important features of network structure might reflect those present in the regional pool and are maintained across spatial scales, as is shown for the degree distribution.

\section{Dispersal in multi-trophic metacommunities}

Dispersal is a key process driving SARs ${ }^{4,47,48}$. Competitive metacommunity models, for instance, have shown that moderate to intermediate levels of dispersal reduce local competitive exclusion, increasing local diversity via colonization-competition tradeoffs ${ }^{48-51}$ or by enhancing source-sink dynamics when resources are heterogeneously distributed in space ${ }^{47,52}$. However, high levels of dispersal would homogenize local communities, leading to regional competitive exclusion and reductions of the overall diversity ${ }^{47,53}$.

The trophic meta-community model also predicts that local diversity increases with dispersal, reducing differences between patches (that is, lower beta-diversity) and leading to a more homogeneous meta-community (Supplementary Fig. 4). The higher the dispersal rate, and thus the lower the spatial beta-diversity, the smaller the effect of increasing area on network properties, because sampling a small number of local communities is enough to capture the structure and composition of the regional community (Supplementary Fig. 5). However, given the absence of direct competitive interactions in our models, both regional and local diversity will increase until they reach the maximum number of species in the regional pool. This observation may differ in the presence of top-down regulation. Our models used a bottom-up sequential food web assembly, with the food chain consistently increasing with the addition of new species. Future explorations of the effect of dispersal on the structure and composition of multi-trophic communities should integrate the trophic constraints used here together with indirect competitive interactions.

A previous study ${ }^{20}$ used a meta-community model to explain the emergence of complex food webs through the linkages between patches provided by omnivorous and generalist species. In our models, the consumer's diet specialization constrains the probability of finding a required resource, and hence, disfavours the presence of specialist consumers in local communities. Given that generalist and omnivorous consumers have more potential resources, they are more likely to persist, which allows for the emergence of network complexity (that is, higher species richness with more links and links per species) in space when local patches are aggregated. Yet, the role of generalists for the spatial scaling of network complexity depends on dispersal. Under dispersal limitation, where a lower number of species coexist locally, generalists are key for the spatial scaling of food web complexity because they are the ones spatially connecting patches. However, in the absence of dispersal limitation, a higher number of species coexist in local communities, increasing the probability of specialists encountering their required prey, and thus, generalists are no longer key contributors to the increase of food web complexity in space. This increase in complexity enhanced by dispersal, on both local and regional scales, might have important implications for the study of the stabilizing effect of space on ecological communities ${ }^{14,17,54}$.

\section{Incorporating spatial scale in comparative studies}

Empirical characterizations of species interaction networks often fail to acknowledge the spatial scale on which these networks are observed. The restricted number of empirical studies that have done so support our theoretical predictions for several NARs. The variation in food chain length with ecosystem size (for example, lake volume) is an example. Although ecosystem productivity can modulate this variation ${ }^{55}$, ecosystem size on its own is a good predictor of food chain length ${ }^{56}$. Our models agree with this empirical observation, showing that MFCL increases with area.

Our predictions of NARs suggest caution must be exercised in comparative studies of network properties. If network properties vary systematically across spatial scales, then comparative network studies that fail to acknowledge the spatial scale on which the study was performed will wrongly estimate the causes of variation of the structure of ecological networks.

The variability observed in food web properties often disappears when species richness is controlled for ${ }^{40-43}$. Then, as area also affects species richness, a key question is to what extent comparative studies addressing variation in network properties need to control additionally for the area sampled, or if the effects of area on network properties are solely driven by richness. In our models, area determines not only the number of species but also their identity based on their feeding traits (that is, more or less generalists) and where they are placed within the food web (that is, across trophic levels). Regardless of area, for a given number of species, we observe differences across models in terms of other network properties (Fig. $5 b, c)$, suggesting that each spatial process has different effects on structuring communities. While network complexity metrics are highly correlated with species (that is, for a given $S$, there is no variation across models), vertical diversity properties are not fully explained by the number of species. For instance, network mean indegree (that is, mean generality; Fig. 5b) shows little variation between models once controlled by the number of species, and this variation disappears when we additionally control by connectance ${ }^{21}$. However, the proportion of species at each trophic level (Fig. 5c) is difficult to predict solely from the number of species, given that each spatial process affects these proportions differently. This suggests that controlling for both species richness and connectance will account for most of the variation in complexity properties across spatial scales, and hence it would suffice in comparative studies, but it would not explain all the variation observed in vertical diversity properties. Therefore, incorporating the spatial scale of sampling in comparative studies would provide additional key information on the scaling of certain network properties.

Moreover, we cannot disregard the effects of habitat size in more complex environments. Our models and the few empirical NARs available mostly concern communities from relatively homogeneous environments. In more heterogeneous landscapes, other processes are at work, such as species sorting (that is, species have different preferences for different habitats within a given area) and priority effects. Intense species sorting is likely to create compartments and result in modular or compartmented webs ${ }^{57,58}$. Food webs are compartmented when interactions between species are either more numerous or stronger within the compartment and few or weak between compartments ${ }^{57,58}$. Our results show very little variation on the modular structure of the communities across spatial scales, but this prediction is likely to be affected when environmental heterogeneity is considered, revealing a potential effect of area on network properties independent of species richness and connectance.

\section{Implications for conservation}

Habitat destruction is the primary cause of the erosion of biodiversity $^{59-61}$. SARs have been extensively used to estimate species loss due to habitat $\operatorname{loss}^{6-8}$. Understanding its effects on the structure of ecological networks is crucial to better preserve ecosystem structure and functioning ${ }^{62-66}$. Our results provide insights into how habitat loss and fragmentation would lead to network simplification, reducing not only species richness, but also - and perhaps more importantly - their interactions. Nonetheless, it is important 
to distinguish the effect of losing habitat (that is, moving across the $x$ axis in Figs. 3 and 5a) from the effect of limiting species dispersal (that is, different dispersal scenarios in Fig. 5a and Supplementary Fig. 5) by, for example, habitat fragmentation. Our results suggest that habitat loss should reduce the number of links per species $(L / S)$ and the proportion of omnivorous species, and shorten food chains (MFCL). Additionally, we observe that fragmented communities with higher dispersal limitation should be less complex across spatial scales with, for example, less species and less links per species. In general, our framework shows that fragmented communities should be expected to be less resistant to habitat loss, showing dramatic changes in food web structure even for small habitat reductions (Fig. 5a and Supplementary Fig. 5).

\section{Limitations and future research}

Species interactions can experience spatial turnover by themselves, correlated or uncorrelated with species composition turnover ${ }^{67}$. Even if two species co-occur in space, they may not interact if the environment is not favourable enough ${ }^{68}$, if one of them is rare $^{69}$, or if they experienced phenological mismatches ${ }^{70}$. Also, we have assumed dispersal constancy across trophic levels. Different scales of movement across trophic levels ${ }^{13,17}$ may also promote variation in network structure across space. Incorporating such processes into theoretical frameworks such as the one presented here could increase the accuracy of our predictions.

Despite the realization that the effect of area on network properties is intimately related to that of richness or connectance, NARs open new possibilities to explore network stability and functioning across spatial scales. Several aspects of food web structure and complexity have been studied locally and related to community stability and functioning, such as the importance of diversity ${ }^{71}$, the presence of stabilizing modules such as the omnivorous loop structures ${ }^{72,73}$, or the predominance of weak interactions ${ }^{74,75}$. Scaling-up in space alters network properties, suggesting that community stability and functioning might also vary across spatial scales. Assessing network structure on different spatial scales can, therefore, provide new insights to analyse and understand community stability and functioning in relation to the different processes that are at play on each spatial scale.

Code availability. Custom code used to develop the theoretical models is available upon request.

Received: 12 May 2017; Accepted: 26 February 2018; Published online: 16 April 2018

\section{References}

1. Arrhenius, O. Species and area. J. Ecol. 9, 95-99 (1921).

2. Rosenzweig, M. L. Species Diversity in Space and Time. (Cambridge Univ. Press: Cambridge, 1995).

3. Lawton, J. H. Are there general laws in ecology? Oikos 84, 177-192 (1999).

4. MacArthur, R. H. \& Wilson, E. O. The Theory of Island Biogeography. (Princeton Univ. Press: Princeton, 1967).

5. Connor, E. F. \& McCoy, E. D. The statistics and biology of the species-area relationship. Am. Nat. 113, 791-833 (1979)

6. Pimm, S. L., Russell, G. J., Gittleman, J. L. \& Brooks, T. M. The future of biodiversity. Science 269, 347-350 (1995)

7. Brooks, T. M. et al. Habitat loss and extinction in the hotspots of biodiversity. Conserv. Biol. 16, 909-923 (2002).

8. Rybicki, J. \& Hanski, I. Species-area relationships and extinctions caused by habitat loss and fragmentation. Ecol. Lett. 16, 27-38 (2013).

9. Holt, R. D., Lawton, J. H., Polis, G. A. \& Martinez, N. D. Trophic rank and the species-area relationship. Ecology 80, 1495-1504 (1999).

10. Roslin, T., Várkonyi, G., Koponen, M., Vikberg, V. \& Nieminen, M. Species-area relationships across four trophic levels - decreasing island size truncates food chains. Ecography 37, 443-453 (2014).

11. Schuldt, A. et al. Multitrophic diversity in a biodiverse forest is highly nonlinear across spatial scales. Nat. Commun. 6, 10169 (2015).

12. Ryberg, W. A. \& Chase, J. M. Predator-dependent species-area relationships. Am. Nat. 170, 636-642 (2007).
13. Rooney, N., McCann, K. S. \& Moore, J. C. A landscape theory for food web architecture. Ecol. Lett. 11, 867-881 (2008).

14. Amarasekare, P. Spatial dynamics of foodwebs. Annu. Rev. Ecol. Evol. Syst. 39, 479-500 (2008).

15. Lafferty, K. D. \& Dunne, J. A. Stochastic ecological network occupancy (SENO) models: a new tool for modeling ecological networks across spatial scales. Theor. Ecol. 3, 123-135 (2010).

16. Gravel, D., Massol, F., Canard, E., Mouillot, D. \& Mouquet, N. Trophic theory of island biogeography. Ecol. Lett. 14, 1010-1016 (2011).

17. McCann, K. S., Rasmussen, J. B. \& Umbanhowar, J. The dynamics of spatially coupled food webs. Ecol. Lett. 8, 513-523 (2005).

18. Brose, U., Ostling, A., Harrison, K. \& Martinez, N. D. Unified spatial scaling of species and their trophic interactions. Nature 428, 167-171 (2004).

19. Thompson, R. M. \& Townsend, C. R. Food-web topology varies with spatial scale in a patchy environment. Ecology 86, 1916-1925 (2005).

20. Pillai, P., Gonzalez, A. \& Loreau, M. Metacommunity theory explains the emergence of food web complexity. Proc. Natl Acad. Sci. USA 108, 19293-19298 (2011)

21. Wood, S. A., Russell, R., Hanson, D., Williams, R. J. \& Dunne, J. A. Effects of spatial scale of sampling on food web structure. Ecol. Evol. 5, 3769-3782 (2015).

22. Gaston, K. J. Global patterns in biodiversity. Nature 405, 220-227 (2000).

23. Cohen, J. E. \& Newman, C. M. When will a large complex system be stable? J. Theor. Biol. 113, 153-156 (1985).

24. Pimm, S. L. Food Webs 1-11 (Univ. Chicago Press, Chicago, 1982).

25. Pimm, S. L., Lawton, J. H. \& Cohen, J. E. Food web patterns and their consequences. Nature 350, 669-674 (1991).

26. Martinez, N. D. Constant connectance in community food webs. Am. Nat. 139, 1208-1218 (1992).

27. Montoya, J. M. \& Sole, R. V. Topological properties of food webs: from real data to community assembly models. Oikos 102, 614-622 (2003).

28. Ings, T. C. et al. Ecological networks - beyond food webs. J. Anim. Ecol. 78, 253-269 (2009)

29. Williams, R. J. \& Martinez, N. D. Simple rules yield complex food webs. Nature 404, 180-183 (2000).

30. Holt, R. D. in The Theory of Island Biogeography Revisited (eds Losos, J. B. \& Ricklefs, R. E.) 143-185 (Princeton Univ. Press, Princeton, 2009).

31. Holt, R. in Food Webs: Integration of Patterns and Dynamics (eds Polis, G. \& Winemiller, K.) 313-323 (Springer, Boston, 1996).

32. Cazelles, K., Mouquet, N., Mouillot, D. \& Gravel, D. On the integration of biotic interaction and environmental constraints at the biogeographical scale. Ecography 39, 921-931 (2016).

33. Hanski, I. Metapopulation Ecology (Oxford Univ. Press, New York, 1999).

34. LandeR., Engen, S. \& Sether, B. E. Stochastic Population Dynamics in Ecology and Conservation. (Oxford Univ. Press: New York, 2003).

35. Piechnik, D. A., Lawler, S. P. \& Martinez, N. D. Food-web assembly during a classic biogeographic study: species' 'trophic breadth' corresponds to colonization order. Oikos 117, 665-674 (2008).

36. Drakare, S., Lennon, J. J. \& Hillebrand, H. The imprint of the geographical, evolutionary and ecological context on species-area relationships. Ecol. Lett. 9, 215-227 (2006).

37. He, F. \& Hubbell, S. P. Species-area relationships always overestimate extinction rates from habitat loss. Nature 473, 368-371 (2011).

38. Soininen, J., McDonald, R. \& Hillebrand, H. The distance decay of similarity in ecological communities. Ecography 30, 3-12 (2007).

39. Garcillán, P. P. \& Ezcurra, E. Biogeographic regions and $\beta$-diversity of woody dryland legumes in the Baja California peninsula. J. Veg. Sci. 14, 859-868 (2003)

40. Bengtsson, J. Confounding variables and independent observations in comparative analyses of food webs. Ecology 75, 1282-1288 (1994).

41. Martinez, N. D. Scale-dependent constraints on food-web structure. Am. Nat. 144, 935-953 (1994).

42. Martinez, N. D. \& Lawton, J. H. Scale and food-web structure: from local to global. Oikos 73, 148-154 (1995).

43. Dunne, J. A. et al. Parasites affect food web structure primarily through increased diversity and complexity. PLoS Biol. 11, e1001579 (2013).

44. Sole, R. V. \& Montoya, M. Complexity and fragility in ecological networks. Proc. R. Soc. Lond. B 268, 2039-2045 (2001).

45. Dunne, J. A., Williams, R. J. \& Martinez, N. D. Food-web structure and network theory: the role of connectance and size. Proc. Natl Acad. Sci. USA 99, 12917-12922 (2002).

46. Montoya, J. M., Pimm, S. L. \& Solé, R. V. Ecological networks and their fragility. Nature 442, 259-264 (2006).

47. Mouquet, N. \& Loreau, M. Community patterns in source-sink metacommunities. Am. Nat. 162, 544-557 (2003).

48. Cadotte, M. W. Dispersal and species diversity: a meta-analysis. Am. Nat. 167, 913-924 (2006)

49. Tilman, D. Competition and biodiversity in spatially structured habitats. Ecology 75, 2-16 (1994) 
50. Cadotte, M. W. Competition-colonization trade-offs and disturbance effects at multiple scales. Ecology 88, 823-829 (2007).

51. Calcagno, V., Mouquet, N., Jarne, P. \& David, P. Coexistence in a metacommunity: the competition-colonization trade-off is not dead. Ecol. Lett. 9, 897-907 (2006)

52. Amarasekare, P. \& Nisbet, R. M. Spatial heterogeneity, source-sink dynamics, and the local coexistence of competing species. Am. Nat. 158, 572-584 (2001).

53. Mouquet, N., Miller, T. E., Daufresne, T. \& Kneitel, J. M. Consequences of varying regional heterogeneity in source-sink metacommunities. Oikos 113 481-488 (2006).

54. Gravel, D., Massol, F. \& Leibold, M. A. Stability and complexity in model meta-ecosystems. Nat. Commun. 7, 12457 (2016).

55. Post, D. M. The long and short of food-chain length. Trends Ecol. Evol. 17, 269-277 (2002).

56. Post, D. M. Testing the productive-space hypothesis: rational and power. Oecologia 153, 973-984 (2007).

57. Pimm, S. L. \& Lawton, J. H. Are food webs divided into compartments? J. Anim. Ecol. 49, 879-898 (1980).

58. Guimerà, R. et al. Origin of compartmentalization in food webs. Ecology $\mathbf{9 1}$, 2941-2951 (2010).

59. Pimm, S. L. \& Raven, P. Biodiversity: extinction by numbers. Nature 403 843-845 (2000).

60. Fahrig, L. Effects of habitat fragmentation on biodiversity. Annu. Rev. Ecol. Evol. Syst. 34, 487-515 (2003).

61. Haddad, N. M. et al. Habitat fragmentation and its lasting impact on Earth's ecosystems. Sci. Adv. 1, e1500052 (2015).

62. Melian, C. J. \& Bascompte, J. Food web structure and habitat loss. Ecol. Lett. 5, 37-46 (2002).

63. Tylianakis, J. M., Tscharntke, T. \& Lewis, O. T. Habitat modification alters the structure of tropical host-parasitoid food webs. Nature 445, 202-205 (2007).

64. Gonzalez, A., Rayfield, B. \& Lindo, Z. The disentangled bank: how loss of habitat fragments and disassembles ecological networks. Am. J. Bot. 98, 503-516 (2011).

65. Cardinale, B. J. et al. Biodiversity loss and its impact on humanity. Nature 486, 59-67 (2012)

66. Fortuna, M. A., Krishna, A. \& Bascompte, J. Habitat loss and the disassembly of mutalistic networks. Oikos 122, 938-942 (2013).

67. Poisot, T., Canard, E., Mouillot, D., Mouquet, N. \& Gravel, D. The dissimilarity of species interaction networks. Ecol. Lett. 15, 1353-1361 (2012).

68. Poisot, T., Bever, J. D., Nemri, A., Thrall, P. H. \& Hochberg, M. E. A conceptual framework for the evolution of ecological specialisation. Ecol. Lett. 14, 841-851 (2011).
69. Canard, E. et al. Emergence of structural patterns in neutral trophic networks. PLoS ONE 7, e38295 (2012).

70. Vázquez, D. P. Degree distribution in plant-animal mutualistic networks: forbidden links or random interactions? Oikos $\mathbf{1 0 8}$ 421-426 (2005)

71. McCann, K. S. The diversity-stability debate. Nature 405, 228-233 (2000).

72. Neutel, A.-M. et al. Reconciling complexity with stability in naturally assembling food webs. Nature 449, 599-602 (2007).

73. Kondoh, M. Building trophic modules into a persistent food web. Proc. Natl Acad. Sci. USA 105, 16631-16635 (2008).

74. McCann, K. S., Hastings, A., Huxel, G. R. \& Fig, T. Weak trophic interactions and the balance of nature. Nature 395, 794-798 (1998).

75. Bascompte, J., Melián, C. J. \& Sala, E. Interaction strength combinations and the overfishing of a marine food web. Proc. Natl Acad. Sci. USA 102, 5443-5447 (2005).

\section{Acknowledgements}

We warmly thank J.-F. Arnoldi, M. Barbier and B. Haegeman for numerous discussions and critical reading of preliminary drafts, which improved the quality of this paper. This work was funded in part through the French Laboratory of Excellence Project 'TULIP' (ANR-10-LABX-41; ANR-11-IDEX-002-02), by a Region Midi-Pyrénées Project (CNRS 121090), by a grant from the Canadian Institute of Ecology and Evolution, and by the NEWFORESTS project.

\section{Author contributions}

N.G., D.G., B.C. and J.M.M. designed research. N.G., M.L., D.G. and J.M.M. conducted research and contributed to the models. N.G., D.G. and J.M.M. wrote the paper, and all authors contributed to editing and discussion.

\section{Competing interests}

The authors declare no competing interests.

\section{Additional information}

Supplementary information is available for this paper at https://doi.org/10.1038/ s41559-018-0517-3

Reprints and permissions information is available at www.nature.com/reprints. Correspondence and requests for materials should be addressed to J.M.M.

Publisher's note: Springer Nature remains neutral with regard to jurisdictional claims in published maps and institutional affiliations. 